\title{
Quantum limit theorems
}

by

Katarzyna Lubnauer (Łódź)

\begin{abstract}
A noncommutative analogue of limit theorems in classical probability theory for distributions of canonical pairs of observables is considered. A complete description of all limit probability operators which are quantum counterparts of the classical infinitely divisible and semistable laws is obtained in the case when scalar norming is generalised to norming by $2 \times 2$ matrices.
\end{abstract}

1. Introduction. The aim of this paper is to find limit probability operators (states) for two cases which in the field of classical probability theory dealing with sums of independent random variables correspond to infinitely divisible and operator semistable laws, respectively. The problem has its origin in C. D. Cushen and R. L. Hudson's work [1], and may be briefly described as follows.

For a canonical pair $(p, q)$ of observables acting on a separable Hilbert space $\mathcal{H}$, let

$$
V(x, y)=e^{i(x p+y q)}, \quad(x, y) \in \mathbb{R}^{2},
$$

be the Weyl operators, and denote by $\left(p_{0}, q_{0}\right)$ the Schrödinger pair of position and momentum operators in $L^{2}(\mathbb{R})$ with its Weyl operator $V_{0}(x, y)$.

The celebrated von Neumann uniqueness theorem asserts that there exist a Hilbert space $\mathcal{K}$ and a unitary mapping

$$
U_{p, q}: \mathcal{H} \rightarrow L^{2}(\mathbb{R}) \otimes \mathcal{K}
$$

such that

$$
V(x, y)=U_{p, q}^{-1}\left(V_{0}(x, y) \otimes \mathbf{1}_{\mathcal{K}}\right) U_{p, q}, \quad(x, y) \in \mathbb{R}^{2} .
$$

Now if $\varrho$ is a state on $\mathbb{B}(\mathcal{H})$, then the map $\varrho_{p, q}$ on $\mathbb{B}\left(L^{2}(\mathbb{R})\right)$ defined as

$$
\varrho_{p, q}(B)=\varrho\left(U_{p, q}^{-1}\left(B \otimes \mathbf{1}_{\mathcal{K}}\right) U_{p, q}\right), \quad B \in \mathbb{B}\left(L^{2}(\mathbb{R})\right),
$$

is a state on $\mathbb{B}\left(L^{2}(\mathbb{R})\right)$, called the distribution of the canonical pair $(p, q)$ in the state $\varrho$.

2000 Mathematics Subject Classification: Primary 46L53; Secondary 60 F05.

Key words and phrases: canonical pairs, probability operators, states, characteristic functions, limit distributions. 
Assume now that $\mathcal{H}$ carries several pairs of canonical observables $\left(p_{1}, q_{1}\right)$, $\ldots,\left(p_{m}, q_{m}\right)$ such that the corresponding Weyl operators

$$
V_{k}(x, y)=e^{i\left(x p_{k}+y q_{k}\right)}, \quad(x, y) \in \mathbb{R}^{2}, k=1, \ldots, m,
$$

pairwise commute. The multidimensional version of the von Neumann theorem asserts that there exist a Hilbert space $\mathcal{R}$ and a unitary mapping

$$
U_{p_{1}, q_{1}, \ldots, p_{m}, q_{m}}: \mathcal{H} \rightarrow \underbrace{L^{2}(\mathbb{R}) \otimes \cdots \otimes L^{2}(\mathbb{R})}_{m \text { times }} \otimes \mathcal{R}
$$

such that

$$
\begin{aligned}
V_{k}(x, y)= & U_{p_{1}, q_{1}, \ldots, p_{m}, q_{m}}^{-1}\left(\mathbf{1}_{L^{2}(\mathbb{R})} \otimes \cdots \otimes V_{0}(x, y) \otimes \cdots\right. \\
& \left.\otimes \mathbf{1}_{L^{2}(\mathbb{R})} \otimes \mathbf{1}_{\mathcal{R}}\right) U_{p_{1}, q_{1}, \ldots, p_{m}, q_{m}}, \quad k=1, \ldots, m .
\end{aligned}
$$

We may thus define the joint distribution $\varrho_{p_{1}, q_{1}, \ldots, p_{m}, q_{m}}$, as a state on $\mathbb{B}\left(L^{2}(\mathbb{R})^{\otimes m}\right)$, by the formula

$$
\begin{aligned}
& \varrho_{p_{1}, q_{1}, \ldots, p_{m}, q_{m}}(B) \\
& \quad=\varrho\left(U_{p_{1}, q_{1}, \ldots, p_{m}, q_{m}}^{-1}\left(B \otimes \mathbf{1}_{\mathcal{R}}\right) U_{p_{1}, q_{1}, \ldots, p_{m}, q_{m}}\right), \quad B \in \mathbb{B}\left(L^{2}(\mathbb{R})^{\otimes m}\right) .
\end{aligned}
$$

The pairs $\left(p_{1}, q_{1}\right), \ldots,\left(p_{m}, q_{m}\right)$ are said to be stochastically independent in the state $\varrho$ if

$$
\varrho_{p_{1}, q_{1}, \ldots, p_{m}, q_{m}}=\varrho_{p_{1}, q_{1}} \otimes \cdots \otimes \varrho_{p_{m}, q_{m}} .
$$

Let now $\left(p_{k n}, q_{k n}\right), k=1, \ldots, k_{n}, n=1,2, \ldots$, be a triangular array of canonical pairs stochastically independent in each row, and let $\left\{A_{k n}: k=\right.$ $\left.1, \ldots, k_{n}, n=1,2, \ldots\right\}$ be a family of $2 \times 2$ matrices

$$
A_{k n}=\left[\begin{array}{ll}
a_{11}^{(k n)} & a_{12}^{(k n)} \\
a_{21}^{(k n)} & a_{22}^{(k n)}
\end{array}\right]
$$

such that the sums

$$
\begin{aligned}
\left(\bar{p}_{n}, \bar{q}_{n}\right) & =\sum_{k=1}^{k_{n}} A_{k n}\left(p_{k n}, q_{k n}\right) \\
& =\sum_{k=1}^{k_{n}}\left(a_{11}^{(k n)} p_{k n}+a_{12}^{(k n)} q_{k n}, a_{21}^{(k n)} p_{k n}+a_{22}^{(k n)} q_{k n}\right) \\
& =\left(\sum_{k=1}^{k_{n}}\left(a_{11}^{(k n)} p_{k n}+a_{12}^{(k n)} q_{k n}\right), \sum_{k=1}^{k_{n}}\left(a_{21}^{(k n)} p_{k n}+a_{22}^{(k n)} q_{k n}\right)\right)
\end{aligned}
$$

are canonical pairs for each $n$. We say that the sequence $\left\{\left(\bar{p}_{n}, \bar{q}_{n}\right): n=\right.$ $1,2, \ldots\}$ converges in distribution in the state $\varrho$ to a limit distribution (state) $\varrho_{0}$ if the states $\varrho_{\bar{p}_{n}, \bar{q}_{n}}$ converge to the state $\varrho_{0}$, i.e.

$$
\varrho_{\bar{p}_{n}, \bar{q}_{n}}(B) \rightarrow \varrho_{0}(B) \quad \text { for each } B \in \mathbb{B}\left(L^{2}(\mathbb{R})\right) .
$$


We want to characterise all the limit distributions $\varrho_{0}$ under one of the following two assumptions:

(i) a natural uniform infinitesimality assumption imposed on the distributions $\varrho_{p_{k n}, q_{k n}}$ and norming matrices $A_{k n}$ (general infinitely divisible case);

(ii) $\varrho_{p_{1 n}, q_{1 n}}=\cdots=\varrho_{p_{k_{n} n}, q_{k_{n} n}}, n=1,2, \ldots$, and $k_{n+1} / k_{n} \rightarrow r \geq 1$ (semistable case).

Observe that in the pioneering work [1] where the central limit theorem is proved, we have a particular case of (ii) above with $k_{n}=n$ and norming matrices $A_{1 n}=\cdots=A_{n n}=(1 / \sqrt{n}) I, I$ being the identity matrix. Also further generalisations of [1] in [13] and [2] follow the same pattern. On the other hand, the general situation described in (i) was considered in [14], where the limit distributions were found for "multidimensional" canonical pairs and scalar norming. This paper may be treated as a generalisation of some results in [14] to matrix norming. Because of the close connection between [14] and our work we have found it convenient to adopt the same setup as in [14]; in particular, the problems as well as their solutions are formulated solely in the language of the so-called characteristic functions, without referring to canonical pairs. The idea of such an approach is as follows. Given a canonical pair $(p, q)$ on $\mathcal{H}$, to each state $\varrho$ on $\mathbb{B}(\mathcal{H})$ there corresponds in a 1-1 way a complex-valued function $\widehat{\varrho}$ on $\mathbb{R}^{2}$ defined by

$$
\widehat{\varrho}(x, y)=\varrho(V(x, y)), \quad(x, y) \in \mathbb{R}^{2},
$$

and called the characteristic function of $\varrho$. In particular, the characteristic function of the distribution $\varrho_{p, q}$ of the canonical pair $(p, q)$ in the state $\varrho$ is

$$
\widehat{\varrho}_{p, q}(x, y)=\varrho_{p, q}\left(V_{0}(x, y)\right), \quad(x, y) \in \mathbb{R}^{2},
$$

and on account of (1.1) and (1.2), we have

$$
\widehat{\varrho}_{p, q}(x, y)=\widehat{\varrho}(x, y) \text {. }
$$

Of paramount importance is the intrinsic characterisation of the characteristic functions as continuous functions taking value 1 at 0 and satisfying some kind of positive-definiteness condition; moreover, to each such function $f$ there corresponds exactly one state $\varrho_{0}$ on $\mathbb{B}\left(L^{2}(\mathbb{R})\right)$ such that $\widehat{\varrho}=f$ (Bochner's theorem). (Observe that $\varrho_{p_{0}, q_{0}}$ is just the distribution of the Schrödinger pair $\left(p_{0}, q_{0}\right)$ in the state $\varrho$.)

Now if

$$
A=\left[\begin{array}{ll}
a_{11} & a_{12} \\
a_{21} & a_{22}
\end{array}\right]
$$


is a $2 \times 2$ matrix such that $\left(p^{\prime}, q^{\prime}\right)=A(p, q)=\left(a_{11} p+a_{12} q, a_{21} p+a_{22} q\right)$ is a canonical pair, an easy calculation yields

$$
\widehat{\varrho}_{p^{\prime}, q^{\prime}}(x, y)=\widehat{\varrho}_{p, q}\left(A^{*}(x, y)\right)=\widehat{\varrho}_{p, q}\left(a_{11} x+a_{21} y, a_{12} x+a_{22} y\right) .
$$

Thus taking into account the above-mentioned description of the class of characteristic functions, we can consider all $2 \times 2$ matrices $A$ (call them admissible) such that for each characteristic function $f$ the function $f \circ A^{*}$ is again a characteristic function.

Performing a similar calculation for the pair $\left(\bar{p}_{n}, \bar{q}_{n}\right)$ defined by (1.4), we obtain, by stochastic independence of $\left(p_{1 n}, q_{1 n}\right), \ldots,\left(p_{k_{n} n}, q_{k_{n} n}\right)$,

$$
\widehat{\varrho}_{\bar{p}_{n}, \bar{q}_{n}}(z)=\widehat{\varrho}_{p_{1 n}, q_{1 n}}\left(A_{1 n}^{*} z\right) \cdots \widehat{\varrho}_{p_{k_{n} n}, q_{k_{n} n}}\left(A_{k_{n} n}^{*} z\right), \quad z=(x, y),
$$

so we can again consider all $k_{n}$-tuples $\left(A_{1}, \ldots, A_{k_{n}}\right)$ of $2 \times 2$ matrices (called admissible tuples) such that for any characteristic functions $f_{1}, \ldots, f_{k_{n}}$ the product $\left(f_{1} \circ A_{1}^{*}\right) \cdots\left(f_{k_{n}} \circ A_{k_{n}}^{*}\right)$ is a characteristic function. To complete the passage from canonical pairs to characteristic functions it remains to invoke the "quantum continuity theorem" proved in [1], which says that the convergence of states on $\mathbb{B}\left(L^{2}(\mathbb{R})\right)$ is equivalent to the pointwise convergence of their characteristic functions. Thus we are led to the problem of finding the class of limits of products of characteristic functions, normed by admissible tuples of $2 \times 2$ matrices.

The "quantum limit theorems" which are investigated in this paper were one of the first subjects of so called "quantum probability"-a field that emerged in the 1970's. Today this field has reached some maturity-an interested reader may consult for instance $[3,9,10]$ to learn more about it.

2. Preliminaries and notation. Let $\Pi$ be the lattice of orthogonal projections in a separable infinite-dimensional Hilbert space $\mathcal{H}$. A noncommutative analogue of a probability measure is a state, i.e. a function $\varrho: \Pi \rightarrow[0,1]$ satisfying

(i) $\varrho(\mathbf{1})=1$,

(ii) $\varrho\left(\sum_{n=1}^{\infty} P_{n}\right)=\sum_{n=1}^{\infty} \varrho\left(P_{n}\right)$ for any sequence $\left\{P_{n}\right\}$ of mutually orthogonal projections, where the series on the left hand side converges in the strong operator topology.

A typical (important!) example of a state is as follows. Let $\varphi$ be a unit vector in $\mathcal{H}$. Define $\varrho$ by

$$
\varrho(P)=\langle P \varphi, \varphi\rangle_{\mathcal{H}}=\|P \varphi\|^{2} .
$$

Such states are called pure (or vector states), and are in a sense basic for quantum mechanics. Notice that pure states have the form $\varrho(P)=\operatorname{tr} P T$, where $T$ is the projection onto the one-dimensional space generated by the 
vector $\varphi$,

$$
T \psi=\langle\psi, \varphi\rangle_{\mathcal{H}}, \quad \psi \in \mathcal{H} .
$$

The celebrated Gleason theorem [4] says that the same is true for every state $\varrho$, i.e. we have

$$
\varrho(P)=\operatorname{tr} P T, \quad P \in \Pi,
$$

where $T$ is a probability operator, i.e. a positive operator on $\mathcal{H}$ of unit trace. This in turn implies that every state has an extension from the lattice of projections $\Pi$ to the algebra $\mathbb{B}(\mathcal{H})$ of all bounded operators on $\mathcal{H}$, given by the formula

$$
\varrho(B)=\operatorname{tr} B T, \quad B \in \mathbb{B}(\mathcal{H}) .
$$

This extension is a bounded positive linear functional on $\mathbb{B}(\mathcal{H})$ of norm one, and the formula above yields a 1-1 correspondence between states and probability operators. In what follows we shall often use the terms "state" and "probability operator" interchangeably. The set of all probability operators on $\mathcal{H}$ will be denoted by $\mathcal{P}$.

Let $z \mapsto V(z)$ be a projective unitary representation of the group $\mathbb{R}^{2}$, satisfying the Weyl-Segal commutation relation

$$
V(z) V\left(z^{\prime}\right)=e^{(i / 2) \Delta\left(z, z^{\prime}\right)} V\left(z+z^{\prime}\right),
$$

where $z, z^{\prime} \in \mathbb{R}^{2}, z=(x, y), z^{\prime}=\left(x^{\prime}, y^{\prime}\right)$, and

$$
\Delta\left(z, z^{\prime}\right)=x y^{\prime}-y x^{\prime} .
$$

By $D$ we shall denote the operator on $\mathbb{R}^{2}$ corresponding to the skew form $\Delta$, i.e.

$$
\left\langle z, D z^{\prime}\right\rangle=\Delta\left(z, z^{\prime}\right) \quad \text { for all } z, z^{\prime} \in \mathbb{R}^{2},
$$

where $\langle\cdot, \cdot\rangle$ is the Euclidean inner product in $\mathbb{R}^{2}$. We know from [5] that the map $T \mapsto \widehat{T}$ (where $T$ is from $\mathcal{L}_{1}$, the set of nuclear operators on $\mathcal{H}$ ) given by

$$
\widehat{T}(z)=\operatorname{tr} T V(z)
$$

extends uniquely to a linear isometric transformation from $\mathcal{L}_{2}$, the set of Hilbert-Schmidt operators on $\mathcal{H}$, onto the space $L^{2}\left(\mathbb{R}^{2}\right)$ of all complex-valued square integrable (with respect to Lebesgue measure) functions $f$ on $\mathbb{R}^{2}$ with the norm

$$
\|f\|_{2}=\left(\frac{1}{2 \pi} \int_{\mathbb{R}^{2}}|f(z)|^{2} d z\right)^{1 / 2} .
$$

The function $\widehat{T}$ is called the characteristic function of the probability operator $T$.

Definition 1. A complex-valued function $f$ on $\mathbb{R}^{2}$ is said to be $\Delta$ positive-definite if for arbitrary complex numbers $c_{1}, \ldots, c_{n}$ and vectors 
$z_{1}, \ldots, z_{n} \in \mathbb{R}^{2}$

$$
\sum_{j, k=1}^{n} c_{j} \bar{c}_{k} f\left(z_{j}-z_{k}\right) e^{(i / 2) \Delta\left(z_{j}, z_{k}\right)} \geq 0 .
$$

"Quantum Bochner's theorem" states that $f=\widehat{T}$ for a certain probability operator $T$ if and only if $f$ is $\Delta$-positive-definite, continuous at the origin and $f(0)=1$ (cf. [5]).

Definition 2. A probability operator $T$ is said to be Gaussian if

$$
\widehat{T}(z)=e^{-\frac{1}{2}\langle q z, z\rangle+i\left\langle z, z_{0}\right\rangle},
$$

where $z_{0} \in \mathbb{R}^{2}$ and $q$ is a nonnegative self-adjoint operator on $\mathbb{R}^{2}$. The operator $q$ is called the Gaussian covariance operator.

A necessary and sufficient condition for $q$ to be the covariance operator for a certain Gaussian probability operator is given by the inequality

$$
\langle q z, z\rangle+\left\langle q z^{\prime}, z^{\prime}\right\rangle \geq \Delta\left(z, z^{\prime}\right)
$$

for all $z, z^{\prime} \in \mathbb{R}^{2}$ (see [5, p. 252]). Let $q=\left[\begin{array}{ll}q_{11} & q_{12} \\ q_{21} & q_{22}\end{array}\right]$ be the representation of $q$ in the canonical basis of $\mathbb{R}^{2}$.

Lemma 1. A symmetric matrix $\left[q_{j k}\right]_{j, k=1,2}$ is the matrix of a Gaussian covariance operator if and only if $q_{11} \geq 0$ and $\operatorname{det}\left[q_{j k}\right] \geq 1 / 4$.

Proof. $q$ is a Gaussian covariance operator if and only if (2.1) holds for $z=(x, y), z^{\prime}=\left(x^{\prime}, y^{\prime}\right)$, that is,

$$
\begin{array}{r}
\left(q_{11} x+q_{12} y\right) x+\left(q_{21} x+q_{22} y\right) y+\left(q_{11} x^{\prime}+q_{12} y^{\prime}\right) x^{\prime}+\left(q_{21} x^{\prime}+q_{22} y^{\prime}\right) y^{\prime} \\
\geq x y^{\prime}-x^{\prime} y
\end{array}
$$

for all $x, y, x^{\prime}, y^{\prime} \in \mathbb{R}$, which is equivalent to the positivity of the matrix

$$
\left[\begin{array}{cccc}
q_{11} & \frac{1}{2}\left(q_{12}+q_{21}\right) & 0 & \frac{1}{2} \\
\frac{1}{2}\left(q_{12}+q_{21}\right) & q_{22} & \frac{1}{2} & 0 \\
0 & \frac{1}{2} & q_{11} & \frac{1}{2}\left(q_{12}+q_{21}\right) \\
-\frac{1}{2} & 0 & \frac{1}{2}\left(q_{12}+q_{21}\right) & q_{22}
\end{array}\right],
$$

or equivalently, for $a=\left(q_{12}+q_{21}\right) / 2$ and $b=q_{11} q_{22}$,

$$
\begin{aligned}
& q_{11} \geq 0, \\
& b-a^{2} \geq 0, \\
& b-\frac{1}{4}-a^{2} \geq 0, \\
& \left(b-\frac{1}{4}-a^{2}\right)^{2} \geq 0,
\end{aligned}
$$


that is,

$$
q_{11} \geq 0, \quad \operatorname{det}\left[q_{j k}\right] \geq 1 / 4 .
$$

Corollary 1. All Gaussian covariance operators are invertible.

The projections belonging to $\mathcal{P}$ will be called pure states. Since every such projection having unit trace must be one-dimensional, this perfectly agrees with the term "pure state" introduced before. A projection $T$ is said to be a ground state if it is a symmetric Gaussian probability operator. The probability operators of the form $Q \circ \mu$, where $Q$ is a ground state and $\mu \in \mathcal{M}\left(\mathbb{R}^{2}\right)$, will be called quasi-classical probability operators. Let $\mathcal{M}\left(\mathbb{R}^{2}\right)$ denote the set of all Borel probability measures on $\mathbb{R}^{2}$. By $\delta_{a}\left(a \in \mathbb{R}^{2}\right)$ we shall denote the probability measure on $\mathbb{R}^{2}$ concentrated at $a$. For any $T \in \mathcal{P}$ and $\mu \in \mathcal{M}\left(\mathbb{R}^{2}\right)$ we put

$$
T \circ \mu=\int_{\mathbb{R}^{2}} V(D z) T V^{*}(D z) \mu(d z),
$$

where the integral is taken in the weak sense. From [14] we know that $\widehat{T \circ \mu}=$ $\widehat{T} \widehat{\mu}$, where $\widehat{\mu}$ denotes the classical characteristic function of $\mu$.

For the sake of completeness we collect below some important results proved in [14].

Proposition 1 ([14, Proposition 1.1]). We have

(i) $(T \circ \mu) \circ \nu=T \circ(\mu \star \nu)$.

(ii) Let $T \in \mathcal{P}$ and $\mu \in \mathcal{M}\left(\mathbb{R}^{2}\right)$. Then $T \circ \mu$ is a projection if and only if $T$ is a projection and $\mu=\delta_{a}$ for a certain vector $a \in \mathbb{R}^{2}$.

Proposition 2 ([14, Proposition 1.2]). A probability operator is Gaussian if and only if it is of the form $Q \circ \gamma$, where $Q$ is a ground state and $\gamma$ is a Gaussian measure on $\mathbb{R}^{2}$.

Proposition 3 ([14, Corollary 1.1]). A pure state is quasi-classical if and only if it is of the form $Q \circ \delta_{a}$, where $Q$ is a ground state and $a \in \mathbb{R}^{2}$.

Let us also note the following important fact which can be found in [5].

Proposition 4. Let $T$ be a probability operator. Then $\widehat{T}^{2}$ is the characteristic function of some $\mu \in \mathcal{M}\left(\mathbb{R}^{2}\right)$.

LEMMA 2. If $\widehat{\mu}=\widehat{T}^{2}$, where $T$ is a probability operator, then $\mu$ is a full measure on $\mathbb{R}^{2}$, i.e. $\mu$ is not concentrated on a line.

Proof. We have $\widehat{T} \in L^{2}\left(\mathbb{R}^{2}\right)$, so $\widehat{\mu}=\widehat{T}^{2} \in L(\mathbb{R})$ and thus $\mu$ is absolutely continuous with respect to Lebesgue measure on $\mathbb{R}^{2}$. It follows that $\mu$ cannot be concentrated on any line. 
Let $\mathcal{B}$ be the subset of $L^{2}\left(\mathbb{R}^{2}\right)$ consisting of all continuous functions vanishing at $\infty$. Then $\mathcal{B}$ contains all $\widehat{T}$ where $T \in \mathcal{P}$ (see [7]). It is shown in [14] that $\mathcal{B}$ is the closure of $\widehat{\mathcal{L}}_{1}$ in the norm $\|f\|=\max _{z \in \mathbb{R}^{2}}|f(z)|+\|f\|_{2}$. Let $\mathfrak{A}$ be the set of all Hilbert-Schmidt operators $T$ for which $\widehat{T} \in \mathcal{B}$. We define the convolution $\star$ in $\mathfrak{A}$ by setting

$$
\widehat{T_{1} \star T_{2}}=\widehat{T}_{1} \widehat{T}_{2} .
$$

Moreover, we put $\|T\|=\|\widehat{T}\|$. Then

$$
\left\|T_{1} \star T_{2}\right\| \leq\left\|T_{1}\right\|\left\|T_{2}\right\|,
$$

and consequently, the convolution algebra $\mathfrak{A}$ is a Banach algebra without unit.

A sequence $\left\{T_{n}\right\}_{n \geq 1}$ of elements of $\mathfrak{A}$ is said to be an approximate unit if for every $S \in \mathfrak{A}, T_{n} \star S \rightarrow S$ in the norm \|\| as $n \rightarrow \infty$. Notice that $\left\{T_{n}\right\}_{n \geq 1}$ forms an approximate unit if and only if $\widehat{T}_{n} \rightarrow 1$ uniformly on every compact subset of $\mathbb{R}^{2}$.

We have the inclusions

$$
\mathcal{P} \subset \mathcal{L}_{1} \subset \mathfrak{A} \subset \mathcal{L}_{2} .
$$

An important fact is that if $T_{n} \in \mathcal{P}$ and $\widehat{T}_{n}$ converges pointwise to a limit function $f$ on $\mathbb{R}^{2}$ which is continuous at the origin, then there exists $T \in \mathcal{P}$ such that $\widehat{T}=f$. This follows from the fact that $\Delta$-positive definiteness is preserved under limits, and from Bochner's theorem.

By means of the isomorphism $T \mapsto \widehat{T}$ between $\mathcal{L}_{2}$ and $L^{2}\left(\mathbb{R}^{2}\right)$, for each $2 \times 2$ matrix $A$ we define a linear transformation $\mathcal{U}_{A}$ of $\mathcal{L}_{2}$ by setting

$$
\left(\widehat{\mathcal{U}_{A} T}\right)(z)=\widehat{T}(A z) \text {. }
$$

It is clear that:

(1) $\mathcal{U}_{A B}=\mathcal{U}_{A} \mathcal{U}_{B}$

(2) $\left\|\mathcal{U}_{A} T\right\|_{2}=(\operatorname{det} A)^{-1 / 2}\|T\|_{2}$ if $A$ is nonsingular,

(3) the algebra $\mathfrak{A}$ is invariant under all transformations $\mathcal{U}_{A}$,

(4) $\mathcal{U}_{A}\left(T_{1} \star T_{2}\right)=\mathcal{U}_{A}\left(T_{1}\right) \star \mathcal{U}_{A}\left(T_{2}\right)$.

Definition 3. An $n$-tuple $\left(A_{1}, \ldots, A_{n}\right)$ of $2 \times 2$ matrices is said to be admissible if

$$
\underset{j=1}{\star} \mathcal{U}_{A_{j}} T_{j} \in \mathcal{P}
$$

for any probability operators $T_{1}, \ldots, T_{n}$. Let $\mathcal{A}_{n}, n=1,2, \ldots$, denote the set of all admissible $n$-tuples.

An $n$-tuple $\left(A_{1}, \ldots, A_{n}\right)$ of $2 \times 2$ matrices is said to be conditionally admissible if (2.2) holds for a given choice $T_{1}, \ldots, T_{n}$ of probability operators. 
EXAMPLE 1. Let

$$
A_{j}=\left[\begin{array}{cc}
1 / \sqrt{n} & 0 \\
0 & 1 / \sqrt{n}
\end{array}\right], \quad j=1, \ldots, n .
$$

Then norming by the matrices $\left(A_{1}, \ldots, A_{n}\right)$ is in fact norming by the scalars $(1 / \sqrt{n}, \ldots, 1 / \sqrt{n})$, and according to the considerations after Proposition 2.5 in [14], $\left(A_{1}, \ldots, A_{n}\right)$ is admissible.

EXAMPLE 2. Let $1 / \sqrt{2 n} \leq \alpha<1 / \sqrt{n}$, and put

$$
A_{j}=\left[\begin{array}{ll}
\alpha & 0 \\
0 & \alpha
\end{array}\right], \quad j=1, \ldots, n .
$$

Let $T_{1}, \ldots, T_{n}$ be Gaussian probability operators with the characteristic functions

$$
\widehat{T}_{1}(z)=\cdots=\widehat{T}_{n}(z)=e^{-\frac{1}{2}\langle z, z\rangle} .
$$

Then

$$
\left(\underset{j=1}{\star} U_{A_{j}} T_{j}\right)^{\wedge}(z)=\prod_{j=1}^{n} e^{-\frac{1}{2}\langle\alpha z, \alpha z\rangle}=e^{-\frac{1}{2} n \alpha^{2}\langle z, z\rangle},
$$

so the covariance operator is $Q=\left[\begin{array}{cc}n \alpha^{2} & 0 \\ 0 & n \alpha^{2}\end{array}\right]$, hence $\operatorname{det} Q=n^{2} \alpha^{4} \geq 1 / 4$, which means that

$$
\stackrel{n}{\star} U_{A_{j}} T_{j} \in \mathcal{P}
$$

thus $\left(A_{1}, \ldots, A_{n}\right)$ is conditionally admissible.

Now let $T_{1}^{\prime}, \ldots, T_{n}^{\prime}$ be Gaussian probability operators with the characteristic functions

$$
\widehat{T}_{1}^{\prime}(z)=\cdots=\widehat{T}_{n}^{\prime}(z)=e^{-\frac{1}{4}\langle z, z\rangle} .
$$

We have

$$
\left(\underset{j=1}{\star} U_{A_{j}} T_{j}^{\prime}\right)^{\wedge}(z)=\prod_{j=1}^{n} e^{-\frac{1}{4}\langle\alpha z, \alpha z\rangle}=e^{-\frac{1}{4} n \alpha^{2}\langle z, z\rangle},
$$

so the covariance operator is $Q^{\prime}=\left[\begin{array}{cc}n \alpha^{2} / 2 & 0 \\ 0 & n \alpha^{2} / 2\end{array}\right]$ and $\operatorname{det} Q^{\prime}=\frac{1}{4} n^{2} \alpha^{4}<1 / 4$, which means that $Q^{\prime}$ is not the covariance operator of a probability operator, i.e.

$$
\stackrel{n}{\star} U_{A_{j}} T_{j}^{\prime} \notin \mathcal{P},
$$

and thus $\left(A_{1}, \ldots, A_{n}\right)$ is not admissible.

A large class of admissible tuples is provided by Lemma 3 below. 
LEMma 3. For an arbitrary $n$-tuple $\left(\varepsilon_{1}, \ldots, \varepsilon_{n}\right)$, where $\varepsilon_{r}=-1$ or 1 , $r=1, \ldots, n$, we have

$$
\left\{\left(A_{1}, \ldots, A_{n}\right): \sum_{r=1}^{n} \varepsilon_{r} \operatorname{det} A_{r}=1\right\} \subset \mathcal{A}_{n} .
$$

Proof. It is easy to check that if $f$ is $\Delta$-positive-definite then so is $\bar{f}$, and consequently, for any $T_{1}, \ldots, T_{n} \in \mathcal{P}, z_{1}, \ldots, z_{m} \in \mathbb{R}^{2}, 2 \times 2$ matrices $A_{1}, \ldots, A_{n}$ and $\varepsilon_{r}=-1$ or $1, r=1, \ldots, n$, the matrices

$$
\left[\widehat{T}_{r}\left(A_{r}\left(z_{j}-z_{k}\right)\right) e^{(i / 2) \varepsilon_{r} \Delta\left(A_{r} z_{j}, A_{r} z_{k}\right)}\right]_{j, k=1}^{m}
$$

are positive-definite.

For $A=\left[\begin{array}{ll}a_{11} & a_{12} \\ a_{21} & a_{22}\end{array}\right]$ we have

$$
\begin{gathered}
\Delta\left(A z_{j}, A z_{k}\right)=\Delta\left(\left[\begin{array}{ll}
a_{11} & a_{12} \\
a_{21} & a_{22}
\end{array}\right]\left[\begin{array}{l}
x_{j} \\
y_{j}
\end{array}\right],\left[\begin{array}{ll}
a_{11} & a_{12} \\
a_{21} & a_{22}
\end{array}\right]\left[\begin{array}{l}
x_{k} \\
y_{k}
\end{array}\right]\right) \\
=\Delta\left(\left(a_{11} x_{j}+a_{12} y_{j}, a_{21} x_{j}+a_{22} y_{j}\right),\left(a_{11} x_{k}+a_{12} y_{k}, a_{21} x_{k}+a_{22} y_{k}\right)\right) \\
=\left(a_{11} x_{j}+a_{12} y_{j}\right)\left(a_{21} x_{k}+a_{22} y_{k}\right)-\left(a_{11} x_{k}+a_{12} y_{k}\right)\left(a_{21} x_{j}+a_{22} y_{j}\right) \\
=a_{11} a_{22}\left(x_{j} y_{k}-x_{k} y_{j}\right)+a_{12} a_{21}\left(y_{j} x_{k}-y_{k} x_{j}\right)=\operatorname{det} A \Delta\left(z_{j}, z_{k}\right) .
\end{gathered}
$$

From the fact that if the matrices

$$
A_{r}=\left[\begin{array}{ll}
a_{11}^{r} & a_{12}^{r} \\
a_{21}^{r} & a_{22}^{r}
\end{array}\right], \quad r=1, \ldots, n,
$$

are positive-definite, then so is their entry-by-entry product

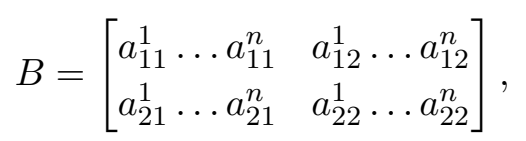

and from the equality above, we deduce that the matrix

$$
\left[\prod_{r=1}^{n} \widehat{T}_{r}\left(A_{r}\left(z_{j}-z_{k}\right)\right) e^{(i / 2) \sum_{r=1}^{n} \varepsilon_{r} \operatorname{det} A_{r} \Delta\left(z_{j}, z_{k}\right)}\right]_{j, k=1}^{m}
$$

is positive-definite. If now $\sum_{r=1}^{n} \varepsilon_{r} \operatorname{det} A_{r}=1$, then $\prod_{r=1}^{n} \widehat{T}_{r}\left(A_{r} z\right)$ is $\Delta$ positive-definite, so

$$
\stackrel{n}{\star} \underset{r=1}{\star} \mathcal{U}_{A_{r}} T_{r} \in \mathcal{P}
$$

which completes the proof.

3. Limit theorems. Let $\left\{T_{k n}\right\},\left\{A_{k n}\right\}, k=1, \ldots, k_{n}, n=1,2, \ldots$, be triangular arrays of probability operators and $2 \times 2$ matrices such that 
$\left(A_{1 n}, \ldots, A_{k_{n} n}\right) \in \mathcal{A}_{k_{n}}$ for $n=1,2, \ldots$ Then

$$
\underset{k=1}{\star k_{n}} \mathcal{U}_{A_{k n}} T_{k n} \in \mathcal{P} \text {. }
$$

The triangular array $\left\{\mathcal{U}_{A_{k n}} T_{k n}: k=1, \ldots, k_{n} ; n=1,2, \ldots\right\}$ of operators from $\mathfrak{A}$ is said to be uniformly infinitesimal if for every choice of $r_{n}, 1 \leq$ $r_{n} \leq k_{n}$, the sequence $\left\{\mathcal{U}_{A_{r_{n} n}} T_{r_{n} n}: n=1,2, \ldots\right\}$ forms an approximate unit in the convolution algebra $\mathfrak{A}$, or equivalently, in terms of characteristic functions,

$$
\lim _{n \rightarrow \infty} \max _{1 \leq k \leq k_{n}}\left|1-\widehat{T}_{k n}\left(A_{k n} z\right)\right|=0
$$

uniformly on every compact subset of $\mathbb{R}^{2}$.

Suppose that for a uniformly infinitesimal triangular array there exists a sequence $\left\{c_{n}\right\}_{n \geq 1}$ of vectors from $\mathbb{R}^{2}$ such that the sequence of probability operators

$$
\left(\underset{k=1}{\star} \mathcal{U}_{A_{k n}} T_{k n}\right) \circ \delta_{c_{n}}
$$

converges to an operator $S$ in $\mathfrak{A}$. Our aim is to characterise the limit probability operators $S$ in two basic cases. Namely, we shall prove

THEOREM 1. The set $D$ of all limit operators of the sequence (3.1) consists of all quasi-classical probability operators $Q \circ \mu$, where $Q$ is a ground state and $\mu$ is an infinitely divisible probability measure from $\mathcal{M}\left(\mathbb{R}^{2}\right)$.

THEOREM 2. The set $D_{1}$ of all limit operators of the sequence (3.1) in the case when $k_{n+1} / k_{n} \rightarrow r \geq 1, A_{1 n}=A_{2 n}=\cdots=A_{n n}, T_{k n}=T$, consists of all Gaussian probability operators.

To prove these theorems we need some facts from the theory of $\sigma$-positivedefinite functions on locally compact groups. To keep the paper as selfcontained as possible, we present the main points of this theory below. For a more detailed account the reader is referred to [11, 12].

In what follows we assume that $G$ is a locally compact group.

Definition 4. Let $I$ be the group of complex numbers $z$ with $|z|=1$. A Borel function $\sigma: G \times G \rightarrow I$ is called a multiplier if

$$
\begin{gathered}
\sigma\left(e, g_{1}\right)=\sigma\left(g_{2}, e\right)=1, \\
\sigma\left(g_{1}, g_{2}\right) \sigma\left(g_{1} g_{2}, g_{3}\right)=\sigma\left(g_{2}, g_{3}\right) \sigma\left(g_{1}, g_{2} g_{3}\right)
\end{gathered}
$$

for all $g_{1}, g_{2}, g_{3} \in G, e$ being the group unit.

Lemma 4. The function $\sigma$ given by the formula

$$
\sigma\left(z_{1}, z_{2}\right)=e^{(i / 2) \Delta\left(z_{1}, z_{2}\right)}, \quad z_{1}, z_{2} \in \mathbb{R}^{2},
$$

is a multiplier on the vector group $\mathbb{R}^{2}$. 
Proof. Let $e=(0,0), z_{1}=\left(x_{1}, y_{1}\right), z_{2}=\left(x_{2}, y_{2}\right), z_{3}=\left(x_{3}, y_{3}\right)$. Then

$$
\sigma\left(e, z_{1}\right)=\sigma\left(z_{2}, e\right)=e^{(i / 2) \Delta\left(e, z_{1}\right)}=e^{(i / 2) \Delta\left(z_{2}, e\right)}=1
$$

and

$$
\begin{aligned}
\sigma\left(z_{1}, z_{2}\right) \sigma\left(z_{1}+z_{2}, z_{3}\right) & =e^{(i / 2)\left[\Delta\left(z_{1}, z_{2}\right)+\Delta\left(z_{1}+z_{2}, z_{3}\right)\right]} \\
& =e^{(i / 2)\left(x_{1} y_{2}-x_{2} y_{1}+x_{1} y_{3}+x_{2} y_{3}-x_{3} y_{1}-x_{3} y_{2}\right)} \\
& =e^{(i / 2)\left[\Delta\left(z_{2}, z_{3}\right)+\Delta\left(z_{1}, z_{2}+z_{3}\right)\right]}=\sigma\left(z_{2}, z_{3}\right) \sigma\left(z_{1}, z_{2}+z_{3}\right),
\end{aligned}
$$

which proves the claim.

Definition 5. Let $\mathcal{U}(\mathcal{H})$ denote the group of all unitary operators on $\mathcal{H}$ with the weak topology. A Borel map $U: g \mapsto U_{g}$ from $G$ into $\mathcal{U}(\mathcal{H})$ satisfying

$$
U_{e}=I, \quad U_{g_{1}} U_{g_{2}}=\sigma\left(g_{1}, g_{2}\right) U_{g_{1} g_{2}}, \quad g_{1}, g_{2} \in G,
$$

is called a $\sigma$-multiplier representation of the group $G$ in $\mathcal{H}$.

Definition 6. A continuous function $s: G \times G \rightarrow \mathbb{C}$ is called an additive multiplier if

(1) $s\left(g_{1}, e\right)=s\left(e, g_{1}\right)=0$,

(2) $s\left(g_{1} g_{2}, g_{3}\right)+s\left(g_{1}, g_{2}\right)=s\left(g_{1}, g_{2} g_{3}\right)+s\left(g_{2}, g_{3}\right)$,

(3) $s\left(g_{1}, g_{2}\right)+s\left(g_{1}^{-1}, g_{2}^{-1}\right)=0$

for all $g_{1}, g_{2}, g_{3} \in G$.

LEMMA 5. The map s given by the formula

$$
s\left(z_{1}, z_{2}\right)=\frac{1}{2} \Delta\left(z_{1}, z_{2}\right), \quad z_{1}, z_{2} \in \mathbb{R}^{2},
$$

is an additive multiplier for the vector group $\mathbb{R}^{2}$.

Proof. Conditions (1), (2) and (3) follow from calculations similar to those in the previous lemma. The continuity of $s$ is obvious.

Definition 7. Let $\sigma$ be a multiplier. A complex-valued function $\varphi$ on $G$ is called $\sigma$-positive-definite if for any complex numbers $c_{1}, \ldots, c_{n}$ and $g_{1}, \ldots, g_{n} \in G$,

$$
\sum_{j, k} c_{j} \bar{c}_{k} \varphi\left(g_{j} g_{k}^{-1}\right) \sigma\left(g_{j}, g_{k}\right) \geq 0 .
$$

Note that for $T \in \mathcal{P}$ the function $\widehat{T}$ is $\sigma$-positive-definite for $\sigma\left(z_{1}, z_{2}\right)=$ $e^{(i / 2) \Delta\left(z_{1}, z_{2}\right)}$.

Definition 8. A triple $(U, \sigma, x)$, where $(U, \sigma)$ is a $\sigma$-multiplier representation of $G$ in $\mathcal{H}$ and $x$ is a unit vector in $\mathcal{H}$, is called a cyclic $\sigma$-multiplier representation if the set $\left\{U_{g} x: g \in G\right\}$ spans $\mathcal{H}$. The function $\varphi(g)=\left\langle U_{g} x, x\right\rangle$ is then called the characteristic function (expectation value) of $(U, \sigma, x)$. 
It is easily seen that the characteristic function $\varphi$ is $\sigma$-positive-definite. Moreover, by a version of the Raykov theorem it follows that for any $\sigma$ positive-definite function $\varphi$ there is a cyclic $\sigma$-multiplier representation $(U, \sigma, x)$ for which $\varphi$ is the characteristic function.

Definition 9. Let $s$ be an additive multiplier on $G \times G$. A continuous function $\psi: G \rightarrow \mathbb{C}$ is called conditionally s-positive-definite if the map $(g, h) \mapsto \psi\left(h^{-1} g\right)+i s\left(h^{-1} g\right)$ is conditionally positive-definite on $G \times G$.

Definition 10. A cyclic $\sigma$-multiplier representation $(U, \sigma, x)$ is called canonical if its characteristic function is nonnegative.

Definition 11. A sequence $\left(U^{(n)}, \sigma_{n}, x_{n}\right)$ of cyclic $\sigma_{n}$-multiplier representations is said to converge to a cyclic $\sigma$-multiplier representation $(U, \sigma, x)$ if the corresponding characteristic functions $\varphi_{n}$ converge pointwise to $\varphi$ and $\sigma_{n}$ converge pointwise to $\sigma$.

Definition 12. A family $\left\{\left(U^{(n, k)}, \sigma_{n k}, x_{n k}\right): k=1, \ldots, k_{n}, n=1,2, \ldots\right\}$ of cyclic $\sigma_{n k}$-multiplier representations is called uniformly infinitesimal if for any $g \in G$,

$$
\lim _{n \rightarrow \infty} \sup _{1 \leq k \leq k_{n}}\left(1-\left|\varphi_{n k}(g)\right|\right)=0
$$

where $\varphi_{n k}$ is the characteristic function of $\left(U^{(n, k)}, \sigma_{n k}, x_{n k}\right)$.

The convolution $\star_{k=1}^{k_{n}}\left(U^{(n, k)}, \sigma_{n k}, x_{n k}\right)$ of the cyclic $\sigma_{n k}$-multiplier representations $\left(U^{(n, k)}, \sigma_{n k}, x_{n k}\right)$ is defined to be the tensor product of the factors. It is clear that the characteristic function of the convolution is the product of the characteristic functions of the factors.

Theorem 3 ([12, Theorem 4.1]). Let $\left\{\left(U^{(n, k)}, \sigma_{n k}, x_{n k}\right): k=1, \ldots, k_{n}\right.$, $n=1,2, \ldots\}$ be a uniformly infinitesimal family of cyclic multiplier representations of a locally compact second countable group $G$ in Hilbert spaces $\mathcal{H}_{n k}$. If the sequence of convolutions

$$
\left(U^{(n)}, \sigma_{n}, x_{n}\right)=\underset{k=1}{\star}\left(U^{(n, k)}, \sigma_{n k}, x_{n k}\right)
$$

converges to a cyclic multiplier representation $(U, \sigma, x)$ of $G$ in a Hilbert space $\mathcal{H}$ with inner product $\langle\cdot, \cdot\rangle$, then the set $G^{\prime}=\left\{g:\left\langle U_{g} x, x\right\rangle \neq 0\right\}$ is an open subgroup of $G$. Furthermore, there exists a real-valued additive multiplier $s$ on $G^{\prime} \times G^{\prime}$ and a conditionally s-positive-definite function $\psi$ on $G$ such that for any $g, h \in G^{\prime}$, $\left\langle U_{g h} x, x\right\rangle\left\{\left\langle U_{g} x, x\right\rangle\left\langle U_{h} x, x\right\rangle\right\}^{-1} \sigma(g, h)=\exp \{\psi(g h)-\psi(g)-\psi(h)+i s(g, h)\}$. 
If the cyclic multiplier representation $(U, \sigma, x)$ is canonical, then we can choose $(s, \psi)$ such that

$$
\left\langle U_{g} x, x\right\rangle=\exp \psi(g) \quad \text { and } \quad \sigma(g, h)=\exp i s(g, h)
$$

for all $g, h \in G^{\prime}$.

Theorem 4 ([11, Theorems 4.3 and 5.3]). Let $G$ be a locally compact abelian second countable group. Each conditionally s-positive-definite continuous function $\psi$ on $G$ satisfies the Lévy-Khinchin formula

$$
\begin{aligned}
\psi(g)= & \int_{\Gamma-\{1\}}[(g, \gamma)-1-\operatorname{im}(g, \gamma)] d F(\gamma) \\
& -\frac{1}{2} \sum_{j, k} q_{j k} \theta_{j}(g) \overline{\theta_{k}(g)}+i a(g)
\end{aligned}
$$

with the Gaussian part

$$
-\frac{1}{2} \sum_{j, k} q_{j k} \theta_{j}(g) \overline{\theta_{k}(g)}
$$

where $\theta_{1}, \theta_{2}, \ldots$ is a sequence of continuous homomorphisms of $G$ into the complex plane, forming a basis of the space $\mathbb{T}(G)$ of homomorphisms from $G$ into $\mathbb{C}$, such that any $\theta \in \mathbb{T}(G)$ can be written as $\theta(g)=\sum_{i=1}^{\infty} \alpha_{i} \theta_{i}(g) ;\left[q_{j k}\right]_{j, k}$ is a positive-definite matrix and $a$ is a real-valued continuous function such that $a(g)=-a(-g)$. Moreover,

$$
s\left(g_{1}, g_{2}\right)=-\frac{1}{2 i} \sum_{j, k} q_{j k}\left[\theta_{j}\left(g_{1}\right) \overline{\theta_{k}\left(g_{2}\right)}-\theta_{j}\left(g_{2}\right) \overline{\theta_{k}\left(g_{1}\right)}\right] .
$$

Note that for $G=\mathbb{R}^{2}$ each homomorphism of $G$ into $\mathbb{C}$ has the form

$$
\theta(x, y)=a x+b y \quad \text { for some } a, b \in \mathbb{C},
$$

so as a basis for $\mathbb{T}(G)$ we can take $\theta_{1}(x, y)=x$ and $\theta_{2}(x, y)=y$.

Consequently, the Gaussian part (3.4) has the form

$$
-\frac{1}{2} \sum_{j, k=1}^{2} q_{j k} \theta_{j}(x, y) \overline{\theta_{k}(x, y)}=-\frac{1}{2}\left[q_{11} x^{2}+\left(q_{12}+q_{21}\right) x y+q_{22} y^{2}\right] .
$$

In what follows we shall only be concerned with $\sigma$ and $s$ as defined by (3.2) and (3.3). In this case $s$ has the form

$$
\begin{aligned}
s\left(\left(x_{1}, y_{1}\right),\left(x_{2}, y_{2}\right)\right)= & -\frac{1}{2 i}\left[q_{11}\left(x_{1} x_{2}-x_{2} x_{1}\right)+q_{12}\left(x_{1} y_{2}-x_{2} y_{1}\right)\right. \\
& \left.+q_{21}\left(x_{2} y_{1}-x_{1} y_{2}\right)+q_{22}\left(y_{1} y_{2}-y_{2} y_{1}\right)\right] \\
= & -\frac{1}{2 i}\left(x_{1} y_{2}-x_{2} y_{1}\right)\left(q_{12}-q_{21}\right) .
\end{aligned}
$$

Now we are in a position to start proving our theorems. 
Proof of Theorem 1. Necessity. Suppose that $T \in \mathcal{D}$. Then

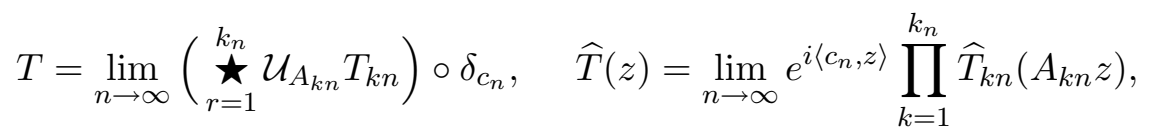

SO

$$
\widehat{T}^{2}(z)=\lim _{n \rightarrow \infty} e^{i\left\langle 2 c_{n}, z\right\rangle} \prod_{k=1}^{k_{n}} \widehat{T}_{k n}^{2}\left(A_{k n} z\right) .
$$

By Proposition 4,

$$
\widehat{\nu}_{k n}=\widehat{T}_{k n}^{2}, \quad \widehat{\nu}=\widehat{T}^{2},
$$

for some probability measures $\nu_{k n}, \nu \in \mathcal{M}\left(\mathbb{R}^{2}\right)$. We have

$$
\widehat{\nu}(z)=\lim _{n \rightarrow \infty} e^{i\left\langle 2 c_{n}, z\right\rangle} \prod_{k=1}^{k_{n}} \widehat{\nu}_{k n}\left(A_{k n} z\right)
$$

and because $\widehat{\nu}_{k n} \circ A_{k n}, k=1, \ldots, k_{n}, n=1,2, \ldots$, are the characteristic functions of the probability measures $A_{k n}^{*} \nu_{k n}$ forming a uniformly infinitesimal array, by the classical limit theorems $\nu$ is infinitely divisible. Moreover, $\nu$ yields the Lévy-Khinchin formula:

$\widehat{\nu}(z)=\exp \left\{i\left\langle m^{\prime}, z\right\rangle-\frac{1}{2}\left\langle q^{\prime} z, z\right\rangle+\int_{\mathbb{R}^{2}-\{0\}}\left[e^{i\langle u, z\rangle}-1-\frac{i\langle u, z\rangle}{1+\|u\|^{2}}\right] M^{\prime}(d u)\right\}$,

where $m^{\prime} \in \mathbb{R}^{2}, q^{\prime}$ is a nonnegative operator on $\mathbb{R}^{2}$, and $M^{\prime}$ is Lévy's measure. Hence

$$
\begin{aligned}
\widehat{T}(z)= & \exp \left\{i\langle m, z\rangle-\frac{1}{2}\langle q z, z\rangle\right. \\
& \left.+\int_{\mathbb{R}^{2}-\{0\}}\left[e^{i\langle u, z\rangle}-1-\frac{i\langle u, z\rangle}{1+\|u\|^{2}}\right] M(d u)\right\},
\end{aligned}
$$

where $m=m^{\prime} / 2, q=q^{\prime} / 2, M^{\prime}=M / 2$.

Let now $\mu$ be Poisson's measure in formula (3.7), i.e.

$$
\widehat{\mu}(z)=\exp \left\{i\langle m, z\rangle+\int_{\mathbb{R}^{2}-\{0\}}\left[e^{i\langle u, z\rangle}-1-\frac{i\langle u, z\rangle}{1+\|u\|^{2}}\right] M(d u)\right\} .
$$

For the measure $\bar{\mu}$ given by

$$
\bar{\mu}(E)=\mu(-E), \quad E \in \mathcal{B}\left(\mathbb{R}^{2}\right),
$$

we have $\widehat{\mu}(z)=\widehat{\mu}(-z)=\overline{\widehat{\mu}(z)}$, so we obtain

$$
\begin{aligned}
e^{-\frac{1}{2}\langle q z, z\rangle}|\widehat{\mu}(z)|^{2} & =e^{-\frac{1}{2}\langle q z, z\rangle} \widehat{\mu}(z) \widehat{\bar{\mu}}(z)=\widehat{T}(z) \widehat{\bar{\mu}}(z) \\
& =\lim _{n \rightarrow \infty} e^{i\left\langle c_{n}, z\right\rangle} \prod_{k=1}^{k_{n}} \widehat{T}_{k n}\left(A_{k n} z\right) \widehat{\bar{\mu}}(z) .
\end{aligned}
$$


Since $\bar{\mu}$ is infinitely divisible, putting $\widehat{\mu}_{k n}(z)=e^{i\left\langle c_{n} / k_{n}, z\right\rangle}[\widehat{\bar{\mu}}(z)]^{1 / k_{n}}$ we obtain from $(3.8)$

$$
|\widehat{\mu}(z)|^{2} e^{\frac{1}{2}\langle q z, z\rangle}=\lim _{n \rightarrow \infty} \prod_{k=1}^{k_{n}}\left[\widehat{T}_{k n}\left(A_{k n} z\right) \widehat{\mu}_{k n}(z)\right] .
$$

As the matrices $A_{k n}$ are admissible, the map $z \mapsto \prod_{k=1}^{k_{n}} \widehat{T}_{k n}\left(A_{k n} z\right)$ is the characteristic function of some probability operator $T_{n}$, so the function

$$
\varphi_{n}(z)=e^{\left\langle c_{n}, z\right\rangle} \prod_{k=1}^{k_{n}} T_{k n}\left(A_{k n} z\right) \widehat{\bar{\mu}}(z)=e^{i\left\langle c_{n}, z\right\rangle} \widehat{\bar{\mu}}(z) \widehat{T}_{n}(z),
$$

being the product of the characteristic function of a probability measure and the characteristic function of a probability operator, is the characteristic function of some probability operator. Consequently, it is $\sigma$-positive-definite, where $\sigma$ is given by (3.2), and hence it is the characteristic function of some cyclic $\sigma_{n}$-multiplier representation of $\mathbb{R}^{2}$ with $\sigma_{n}=\sigma$. The limit function $z \mapsto|\widehat{\mu}(z)|^{2} e^{-\frac{1}{2}\langle q z, z\rangle}$ is also $\sigma$-positive-definite, so it is the characteristic function of some cyclic $\sigma$-multiplier representation of $\mathbb{R}^{2}$. Moreover, it is positive-definite, so by Theorem 3 we obtain

$$
|\widehat{\mu}(z)|^{2} e^{-(i / 2)\langle q z, z\rangle}=e^{\psi(z)},
$$

where $\psi$ is a conditionally $s$-positive-definite function. Moreover,

$$
\begin{aligned}
|\widehat{\mu}(z)|^{2}=\widehat{\mu}(z) \widehat{\mu}(-z)= & \exp \left\{\int_{\mathbb{R}^{2}-\{0\}}\left[e^{i\langle u, z\rangle}-1-\frac{i\langle u, z\rangle}{1+\|u\|^{2}}\right] M(d u)\right. \\
& \left.+\int_{\mathbb{R}^{2}-\{0\}}\left[e^{i\langle-u, z\rangle}-1-\frac{i\langle-u, z\rangle}{1+\|u\|^{2}}\right] M(d u)\right\} \\
= & \exp \left\{\int_{\mathbb{R}^{2}-\{0\}}\left[e^{i\langle u, z\rangle}-1-\frac{i\langle u, z\rangle}{1+\|u\|^{2}}\right] M(d u)\right. \\
& \left.+\int_{\mathbb{R}^{2}-\{0\}}\left[e^{i\langle u, z\rangle}-1-\frac{i\langle u, z\rangle}{1+\|u\|^{2}}\right] \bar{M}(d u)\right\},
\end{aligned}
$$

where $\bar{M}(E)=M(-E)$. Putting $M_{1}=M+\bar{M}$ we get

$$
\begin{aligned}
|\widehat{\mu}(z)|^{2} & =\exp \left\{\int_{\mathbb{R}^{2}-\{0\}}\left[e^{i\langle u, z\rangle}-1-\frac{i\langle u, z\rangle}{1+\|u\|^{2}}\right] M_{1}(d u)\right\} \\
& =\exp \left\{\int_{\mathbb{R}^{2}-\{0\}}[\cos \langle u, z\rangle-1] M_{1}(d u)\right\} .
\end{aligned}
$$


From (3.9) and (3.10) we finally obtain

$$
\psi(z)=\int_{\mathbb{R}^{2}-\{0\}}[\cos \langle u, z\rangle-1] M_{1}(d u)-\frac{1}{2}\langle q z, z\rangle .
$$

Since $\psi$ is $s$-conditionally positive-definite, its Gaussian part has the form given by (3.5). The (complex) matrix $\left[q_{j k}\right]$ is positive-definite, thus

$$
q_{11}, q_{22} \geq 0, \quad q_{12}=\bar{q}_{21}, \quad \operatorname{det}\left[q_{j k}\right] \geq 0 .
$$

Put

$$
\left[q_{j k}^{*}\right]=\left[\begin{array}{cc}
q_{11} & \left(q_{12}+q_{21}\right) / 2 \\
\left(q_{12}+q_{21}\right) / 2 & q_{22}
\end{array}\right] .
$$

This is a real matrix with

$$
\begin{aligned}
\operatorname{det}\left[q_{j k}^{*}\right] & =q_{11}^{*} q_{22}^{*}-q_{12}^{*} q_{21}^{*}=q_{11} q_{22}-q_{12} q_{21}-\frac{1}{4}\left(q_{12}-q_{21}\right)^{2} \\
& =\operatorname{det}\left[q_{j k}\right]-\frac{1}{4}\left(q_{12}-q_{21}\right)^{2} \geq-\frac{1}{4}\left(q_{12}-q_{21}\right)^{2},
\end{aligned}
$$

since $\operatorname{det}\left[q_{j k}\right] \geq 0$.

Now taking into account the connection between $\sigma$ and $\Delta$ given by formulae (3.3) and (3.6), we get the equality

$$
-\frac{1}{2 i}\left(x_{1} y_{2}-x_{2} y_{1}\right)\left(q_{12}-q_{21}\right)=\frac{1}{2}\left(x_{1} y_{2}-x_{2} y_{1}\right),
$$

and thus $q_{12}-q_{21}=i$. Consequently, from inequality (3.11) we obtain

$$
\operatorname{det}\left[q_{j k}^{*}\right] \geq 1 / 4 \text {. }
$$

From Lemma 1 it follows that $\left[q_{j k}^{*}\right]$ is the matrix of a Gaussian covariance operator, so the Gaussian part of the characteristic function of $T$ is nonzero.

Sufficiency. This follows from [14] where it is shown that we can take the norming operators $A_{n}$ to be multiples of the identity operator.

Proof of Theorem 2. Necessity. Let

$$
T=\lim _{n \rightarrow \infty}\left(\underset{k=1}{k_{n}} \mathcal{U}_{A_{k n}} T_{k n}\right) \circ \delta_{c_{n}}
$$

where $A_{1 n}=A_{2 n}=\cdots=A_{k_{n} n}, T_{k n}=S$ and $k_{n+1} / k_{n} \rightarrow r \geq 1$.

Putting $\widehat{\nu}(z)=\widehat{S}^{2}(z)=\widehat{T}_{k n}^{2}$ and $\widehat{\mu}(z)=\lim _{n \rightarrow \infty} \prod_{k=1}^{k_{n}} \widehat{\nu}\left(A_{k n} z\right) e^{i\left\langle c_{n}, z\right\rangle}$ we have $\widehat{\mu}(z)=\widehat{T}^{2}(z)$, and $\mu$ is an operator semistable probability measure (see $[6,8]$ for a more detailed description of such measures).

By Theorem 1, $T$ has nonzero Gaussian part, and from Corollary 1 it follows that this Gaussian part is full on $\mathbb{R}^{2}$. Now by [6, Theorem 1.1], we conclude that $\mu$ and thus $T$ must be Gaussian.

Sufficiency. This is proved as Theorem 3.3 in [14] for $s=1, k_{n}=n$ and $A_{1 n}=A_{2 n}=\cdots=A_{n n}=\left[\begin{array}{cc}1 / \sqrt{n} & 0 \\ 0 & 1 / \sqrt{n}\end{array}\right]$. 
REMARK. Observe that Theorems 1 and 2 can be slightly generalised. Namely, we may consider limits of sequences as in (3.1) assuming only that the norming matrices $A_{1 n}, \ldots, A_{k_{n} n}$ are conditionally admissible. It follows from the proofs of Theorems 1 and 2 that in this case we obtain the same limit classes.

\section{References}

[1] C. D. Cushen and R. L. Hudson, A quantum mechanical central limit theorem, J. Appl. Probab. 8 (1971), 454-469.

[2] E. Czkwianianc, Symmetric semistable measures on $\mathbb{R}^{n}$ and symmetric semistable distribution operators in quantum mechanics, Rep. Math. Phys. 17 (1980), 89-99.

[3] E. B. Davies, Quantum Theory of Open Systems, Academic Press, London, 1976.

[4] A. M. Gleason, Measures on the closed subspaces of a Hilbert space, J. Math. Mech. 6 (1957), 885-894.

[5] A. S. Holevo, Probabilistic and Statistical Aspects of Quantum Theory, Nauka, Moscow, 1980 (in Russian).

[6] R. Jajte, Semi-stable probability measures on $\mathbb{R}^{N}$, Studia Math. 61 (1977), 29-39.

[7] L. H. Loomis, An Introduction to Abstract Harmonic Analysis, Van Nostrand, Toronto, 1953.

[8] A. Euczak, Operator semi-stable probability measures on $\mathbb{R}^{N}$, Colloq. Math. 45 (1981), 287-300.

[9] P. A. Meyer, Quantum Probability for Probabilists, Lecture Notes in Math. 1538, Springer, Berlin, 1995.

[10] K. R. Parthasarathy, An Introduction to Quantum Stochastic Calculus, Birkhäuser, Basel, 1992.

[11] K. R. Parthasarathy and K. Schmidt, Infinitely divisible projective representations, cocycles and Lévy-Khinchine-Araki formula on locally compact groups, preprint, Univ. of Manchester, 1970.

[12] K. Schmidt, Limits of uniformly infinitesimal families of projective representations of locally compact groups, Math. Ann. 192 (1971), 107-118.

[13] K. Urbanik, Stable symmetric probability laws in quantum mechanics, Bull. Acad. Polon. Sci. Sér. Sci. Math. Astronom. Phys. 23 (1975), 799-806.

[14] —, Non-commutative probability limit theorems, Studia Math. 78(1984), 59-75.

Faculty of Mathematics

Łódź University

S. Banacha 22

90-238 Łódź, Poland

E-mail: lubnauer@math.uni.lodz.pl

Received May 15, 2002

Revised version January 12, 2004 